\title{
Adaptive Control of a Production-Inventory Model with Uncertain Deterioration Rate
}

\author{
Fawzy Bukhari \\ Department of Statistics and Operations Research, College of Science, King Saud University, Riyadh, Saudi Arabia \\ E-mail: fbukhari@ksu.edu.sa \\ Received July 6, 2011; revised July 22, 2011; accepted July 29, 2011
}

\begin{abstract}
This paper studied a continuous-time model of a production maintenance system in which a manufacturing firm produces a single product selling some and stocking the remaining. The problem of adaptive control of a production-maintenance system with unknown deterioration has been presented. Using Liapunov technique, the production rate and updating rule of deterioration rate are derived as non-linear functions of inventory level perturbation. Numerical analysis for the system has been presented for a set of parameter values and demand rate.
\end{abstract}

Keywords: Production Inventory System, Adaptive Control, Liapunov Technique

\section{Introduction}

Applications of optimal control theory to management science, in general, and to production planning, in particular, are proving to be quite fruitful; see for example Sethi and Thompson [1]. Naturally, with the optimal control theory, optimal control techniques came to be applied to production planning problems and others. For example, Dobos [2] have discussed the problem of a reverse logistics system with special structure. The problem was presented as an optimal control problem with two state variables and with three control variables with an objective to minimize the sum of the quadratic deviation from the goal values. Nahmias [3], Porter and Taylor [4], Raafat [5], and Khemlnitsky and Gerchak [6] have discussed the problems of production inventory systems with inventory levels dependant on demand rate, the problem of modal control of production inventory systems, and inventory systems with deteriorating items. Caldwell [7], Aseltine, Mancini and Sartune [8], Landau [9], and Narendra and Annaswamy [10] have solved many problems by using the adaptive control of linear and non linear dynamical systems using a feedback approach. El-Gohary and Al-Ruzaiza [11] have studied the adaptive control of a continuous time three species prey predator populations. They have derived the nonlinear feedback control inputs asymptotically stabilized the system about its steady states. El-Gohary and Yassen [12] have used the adaptive control and synchronization pro- cedures to the coupled dynamo system with unknown parameters. Based on the Liapunov stability technique, an adaptive control laws are derived such that the coupled dynamo system is asymptotically stable and the two identical dynamo systems are asymptotically synchronized. Also, the update rules of the unknown parameters are derived. Tadj, Sarhan and El-Gohary [13] have discussed the optimal control of an inventory system with ameliorating and deteriorating items. Different cases for the difference between the ameliorating and deteriorating items are considered. Foul, Tadj, and Hedjar [14] have studied the problem of adaptive control of a production inventory system in which a manufacturing firm produces a single product selling some and stocking the remaining. Model reference adaptive control with feedback is applied to track the output of the system toward the inventory goal level.

The paper studies a production-inventory model which produces a single item with a certain production rate and seeks an adaptive actual production and inventory rates. This model has a dynamic nature and an adaptive control approach seems particularly well suited to achieve its goal level and production rate. Also, the unknown deterioration rate will be derived from the conditions of asymptotic stabilization about the steady-state.

The rest of the paper is organized as follows. Following this introduction, the mathematical model is introduced in Section 2. In Section 3, the problem of adaptive control of the production inventory system with un- 
known deterioration rate is discussed. Also the desired production rate and updating rule of the deterioration rate are derived from the conditions of the asymptotic stability of the system. In Section 4, we present some illustrative examples. The numerical solution of the controlled model is presented. Section 5 presents a conclusion of the paper.

\section{Mathematical Model}

Consider a manufacturing system that produces a single product, selling some units and adding others to inventory. We start by writing the differential equation models. These can be conveniently formulated in terms of Moreover, the following variables and parameters are used:

$I(t)$ : the inventory level at time $t$,

$P_{a}(t)$ : the actual production rate at time $t$,

$D(t)$ : the demand rate at time $t$,

$u(t)$ : the desired production rate at time $t$,

$\theta$ : the deterioration rate,

$\alpha$ : the inverse of exponential delay time,

$\bar{I}$ : the inventory level at the steady state,

$\bar{P}_{a}$ : the actual production rate at steady state,

$\bar{D}$ : the demand rate at steady state.

All functions are assumed to be nonnegative, continuous and differentiable. Assume that at the time instant $t$ the demand occurs at rate $D(t)$, the actual production at rate $P_{a}(t)$, and the actual production rate responds to the desired production rate with an exponential time-delay of $\alpha^{-1}$, it follows that the inventory level $I(t)$ and the actual production rate $P_{a}(t)$ evolve according to the following first order differential equations:

$$
\left.\begin{array}{c}
\dot{I}(t)=P_{a}(t)-\hat{\theta}(t) I(t)-D(t), \\
\dot{P}_{a}(t)=\alpha\left(u(t)-P_{a}(t)\right),
\end{array}\right\}
$$

where, the "dot" means the differentiation with respect to time and the initial state is $I(0)=I_{0}, P_{a}(0)=P_{a}$. This balance equations were also used by Porter and Taylor (1972), Bradshaw and Porter (1975), and Riddalls and Bennett (2001) without deterioration rate $\theta$. The steady-states of this system can be derive by setting both $\dot{I}(t)$ and $\dot{P}_{a}(t)$ equal to zero. That is:

$$
\bar{I}=\theta^{-1}\left(\bar{p}_{a}-\bar{D}\right), \bar{p}_{a}=\bar{u}
$$

Next, in what follows we will discuss the adaptive control of the production inventory model with unknown deterioration rate using Liapunov technique.

\section{Adaptive Control Problem}

In this section, we will discuss in details the adaptive control problem of the reference model. The rates of demand and desired production, and updating rule of deterioration rate will be derived from the condition of asymptotic stability of the reference model. To study the adaptive control problem we start by considering the values of required inventory goal level, and actual production goal rate as the steady-state solution of the reference model Equation (1). We consider the adaptive system as follows:

$$
\left.\begin{array}{c}
\dot{I}(t)=P_{a}(t)-\hat{\theta}(t) I(t)-D(t), \\
\dot{P}_{a}(t)=\alpha\left(u(t)-P_{a}(t)\right),
\end{array}\right\}
$$

where, $\hat{\theta}(t)$ is the dynamic estimator of the unknown deterioration rate $\theta$. Following, we assume that $I(t)=\bar{I}$, $P_{a}(t)=\bar{P}_{a}, u(t)=\bar{u}, D(t)=\bar{D}$, and $\hat{\theta}(t)=\theta$ is a special solution for the system Equation (3). The equations of perturbed states of the reference system about its steady states can be derived by introducing the following new variables:

$$
\left.\begin{array}{c}
\xi_{1}(t)=I(t)-\bar{I}, \xi_{2}(t)=P_{a}(t)-\bar{P}_{a}, \\
v(t)=u(t)-\bar{u}, d(t)=D(t)-\bar{D}, \eta(t)=\hat{\theta}(t)-\theta,
\end{array}\right\}
$$

Substituting from Equation (4) into Equation (3) we get the following perturbed system:

$$
\left.\begin{array}{c}
\dot{\xi}_{1}(t)=\xi_{2}(t)-\eta(t) \xi_{1}(t)-\theta \xi_{1}(t)-\bar{I} \eta(t)-d(t), \\
\dot{\xi}_{2}(t)=\alpha\left[v(t)-\xi_{2}(t)\right],
\end{array}\right\}
$$

The system Equation (5) will be used to study the adaptive control problem using the Liapunov technique for the system of Equation (4). The following theorem gives the desired production rate and updating rule for deterioration rate ensure the asymptotic stability of the reference production inventory model with uncertain deterioration rate.

\section{Theorem 1}

If the perturbations of both desired production rate and updating rule of the deterioration rate are given by:

$$
v(t)=-\left(\xi_{1}(t)+k \xi_{2}(t)\right) / \alpha
$$

and

$$
\dot{\eta}(t)=\xi_{1}^{2}(t)+\bar{I} \xi_{1}(t)-m \eta(t)
$$

where, $k$, and $m$ are positive real control gains parameters. Then the special solution $\xi_{i}(t)=0,(i=1,2), v(t)=0$, and $\eta(t)=0$ of the systems Equations (5) and (7) is 
asymptotically stable in Liapunov sense if the demand rate is linear function of the perturbation of the inventory level or the actual production rate. Otherwise, the asymptotic stability of the solution requires further mathematical analysis.

Proof: The proof of this theorem based on the selection of a suitable Liapunov function for the system consists of the two combined systems Equations (5) and (7).

Assume this function has the form:

$$
\Phi\left(\xi_{1}, \xi_{2}, \eta\right)=\sum_{i=1}^{2} \xi_{i}^{2}(t)+\eta^{2}(t)
$$

This function is a positive definite function of the variables $\xi_{i}(t),(i=1,2)$, and $\eta(t)$. The total time derivative of the Liapunov function Equation (8) along the trajectory of the systems Equations (5) and (7) give:

$$
\dot{\Phi}=-\left[\theta \xi_{1}^{2}(t)+(\alpha+k) \xi_{2}^{2}(t)+m \eta^{2}(t)\right]-d(t) \xi_{1}(t)
$$

Since $\alpha+k>0, m>0$, the sign of $\dot{\Phi}$ depends upon the perturbation of the demand rate. In this paper, we will consider the following two possibilities which are: $d(t)=f(t) \xi_{1}(t)$ or $d(t)=g(t) \xi_{2}(t)$. Hence, the total time derivative of the Liapunov function takes the form:

$$
\dot{\Phi}=\left\{\begin{array}{c}
-(\theta+f(t)) \xi_{1}^{2}(t)-(\alpha+k) \xi_{2}^{2}(t)-m \eta^{2}(t), \\
-\theta \xi_{1}^{2}(t)-(\alpha+k) \xi_{2}^{2}(t)-m \eta^{2}(t)-g(t) \xi_{1}(t) \xi_{2}(t),
\end{array}\right.
$$

So, the special solution: $\xi_{i}(t)=0,(i=1,2), \quad v(t)=0$, and $\eta(t)=0$ is asymptotically stable in the Liapunov sense if: $g<2 \sqrt{\theta(\alpha+k)}$ which completes the proof.

Now, substituting the adaptive controlled desired production rate and updating rule of the deterioration rate Equation (5) into Equation (4) we get the following controlled system:

$$
\left.\begin{array}{c}
\dot{\xi}_{1}(t)=\xi_{2}(t)-\eta(t) \xi_{1}(t)-\theta \xi_{1}(t)-\bar{I} \eta(t)-d(t), \\
\dot{\xi}_{2}(t)=-\xi_{1}(t)-k \xi_{2}(t)-\alpha \xi_{2}(t), \\
\dot{\eta}(t)=\bar{I} \xi_{1}(t)+\xi_{1}^{2}(t)-m \eta(t),
\end{array}\right\}
$$

The above system is used to study the time evolution of inventory levels and dynamic estimators of deterioration rates. It appears from Equation (11) that the analytical solution of the system is difficult to derive since it is non-linear and therefore we solve it numerically in the next section.

\section{Numerical Solution}

The objective of this section is to study the numerical solution of the problem to determine an adaptive control strategy for a production inventory model with unknown deterioration rate. The numerical solution algorithm is based on numerical integration of the system using the Runge-Kutta method. This section displays graphically the numerical integration of system Equation (11) of differential equations for a set of parameter values.

\subsection{Example 1}

In this example we will graphically display the system behavior subjected to linear demand rate of the inventory perturbation. The following set of parameter values is assumed:

\begin{tabular}{ccccccc}
\hline Parameter & $k$ & $m$ & $f$ & $\theta$ & $\bar{I}$ & $\alpha$ \\
\hline Value & 3 & 3 & 2 & 0.05 & 50 & 0.2 \\
\hline
\end{tabular}

With the following values of initial perturbations of inventory level, actual production rate, and deterioration rate: $\xi_{1}(0)=40, \xi_{2}(0)=15, \eta(0)=0.02$ respectively. The numerical results are illustrated in Figures 1(a)-(d) as follows:

Figures 1(a)-(c) have indicated that both the perturbations of inventory level and deterioration rate are damping oscillate about their goal values. While Figure 1(b) indicates that the actual production rate perturbation exponentially tends to its goal value. Finally, Figure 1(d) shows that the desired production rate exponentially oscillates about its goal value.

\subsection{Example 2}

In this example we will graphically display the system behavior subjected to linear demand rate of the actual production rate perturbation. The following set of parameter values is assumed:

\begin{tabular}{ccccccc}
\hline Parameter & $k$ & $m$ & $f$ & $\theta$ & $\bar{I}$ & $\alpha$ \\
\hline Value & 0.05 & 0.01 & 0.2 & 0.05 & 200 & 5 \\
\hline
\end{tabular}

With the following values of initial perturbations of inventory level, actual production rate, and deterioration rate: $\xi_{1}(0)=50, \xi_{2}(0)=15, \eta(0)=0.02$ respectively.

The numerical results are illustrated in Figures 2(a)-(d) as follows:

Figures 2(a)-(d) have indicates that the perturbations of inventory level, deterioration rate, and desired production rate are damping oscillate about their goal values. While Figure 2(b) indicates that the actual production rate perturbation exponentially tends to its goal value. 


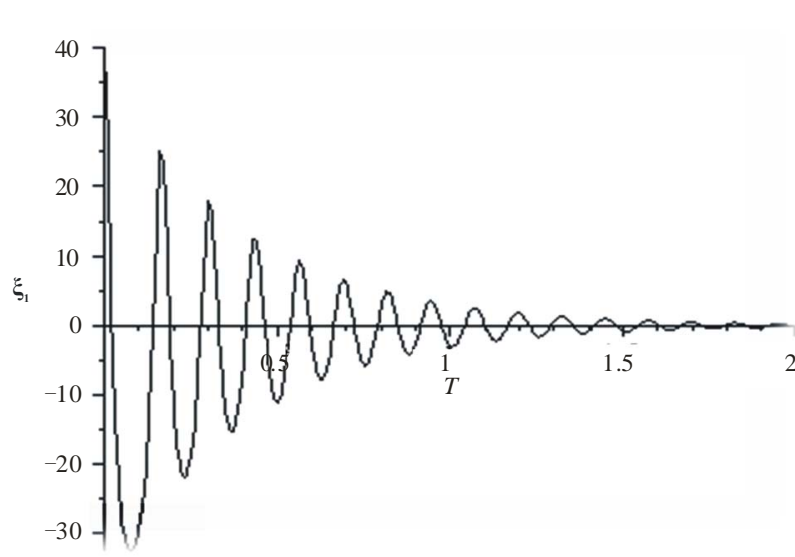

(a)

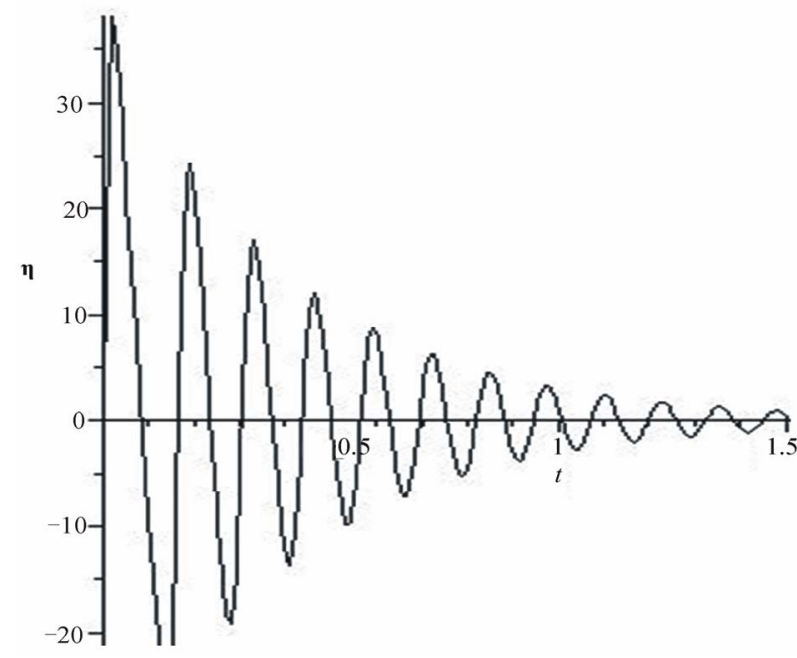

(c)

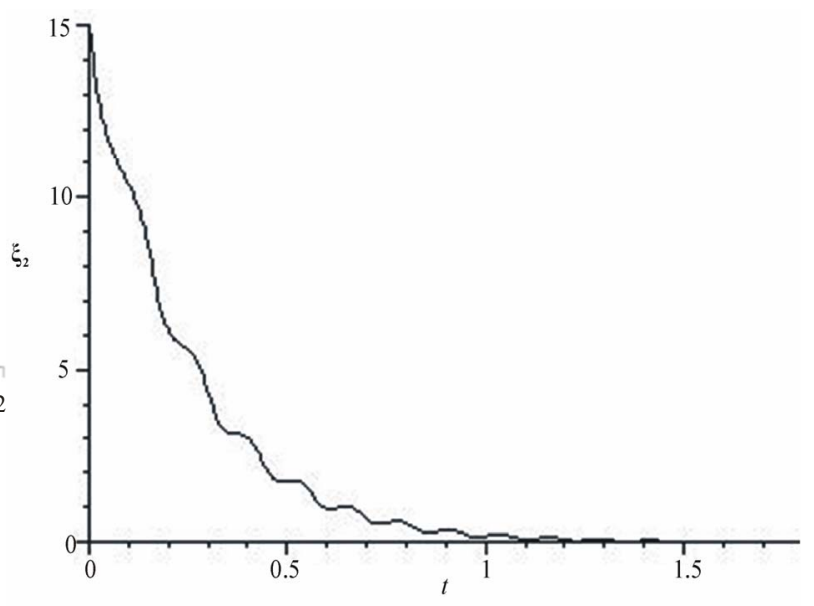

(b)

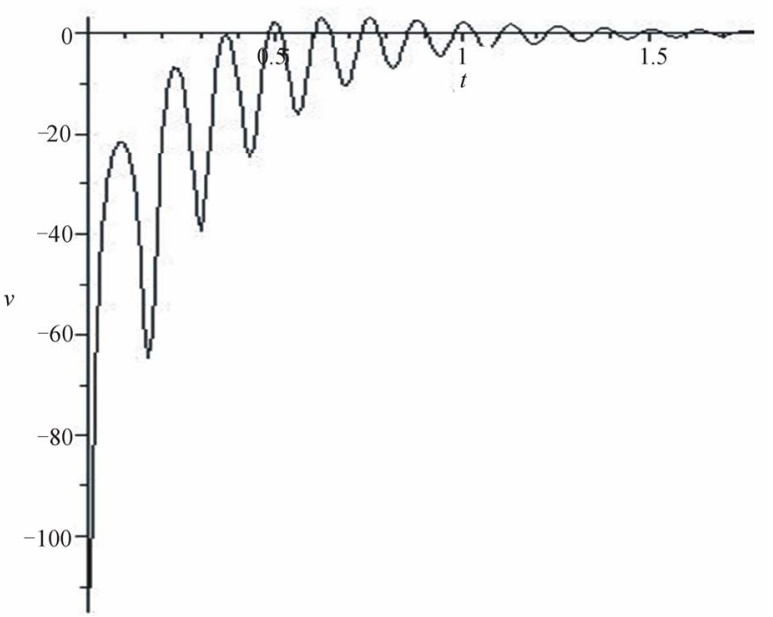

(d)

Figure 1. (a) Perturbation of inventory level; (b) Perturbation of actual production rate; (c) Perturbation of deterioration rate; (d) Perturbation of desired production rate.

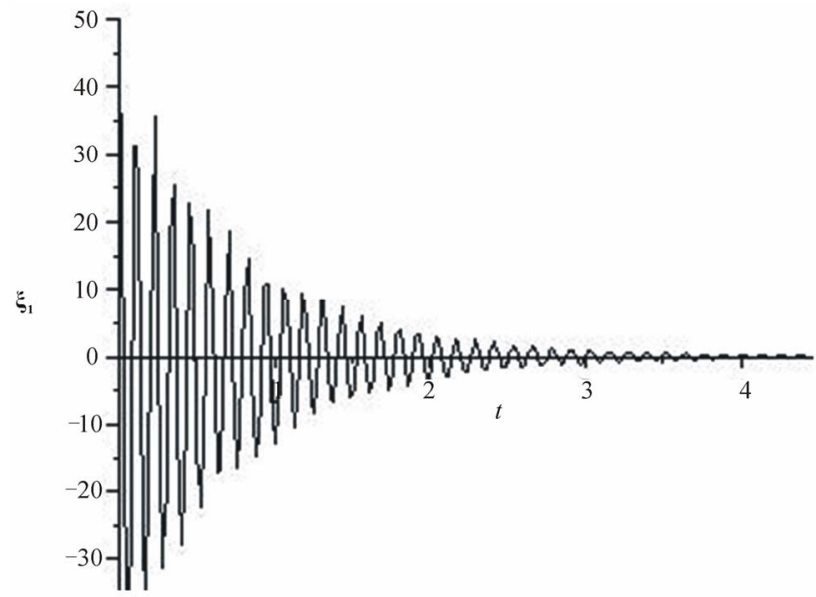

(a)

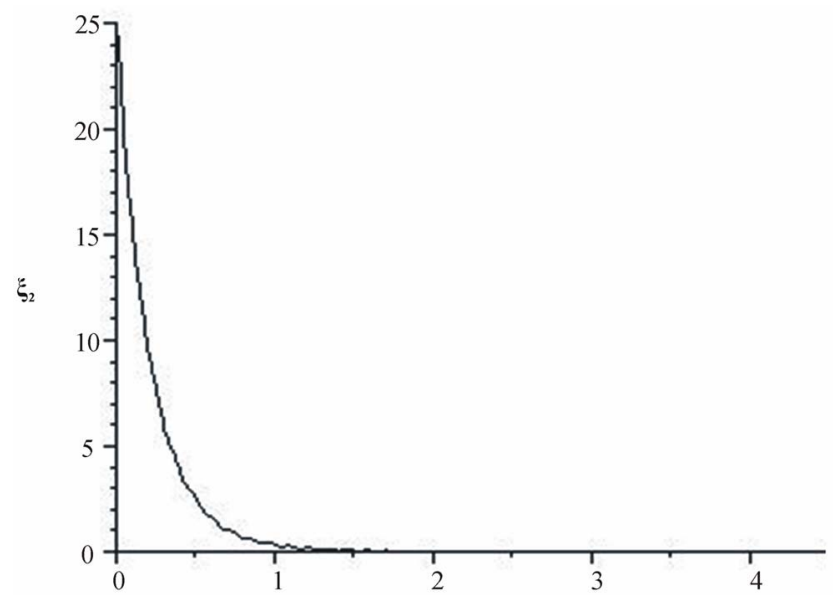

(b) 


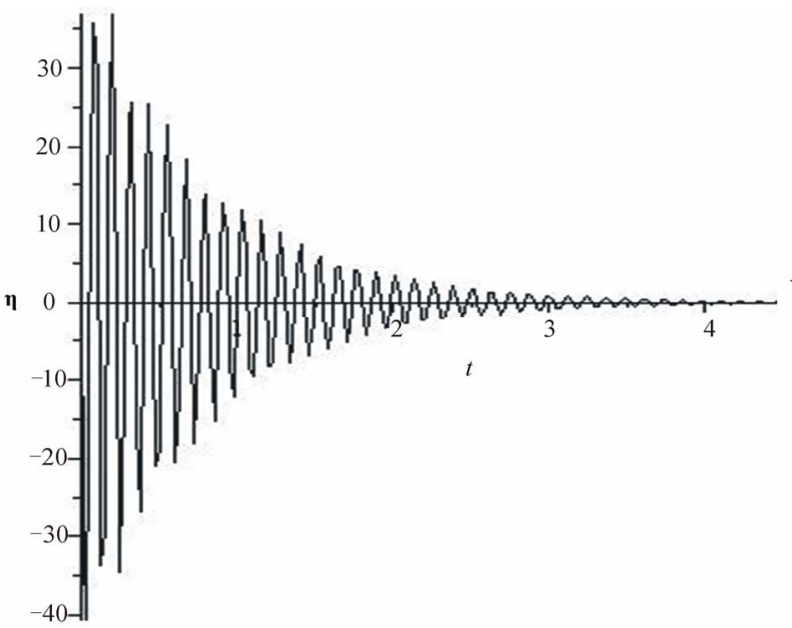

(c)

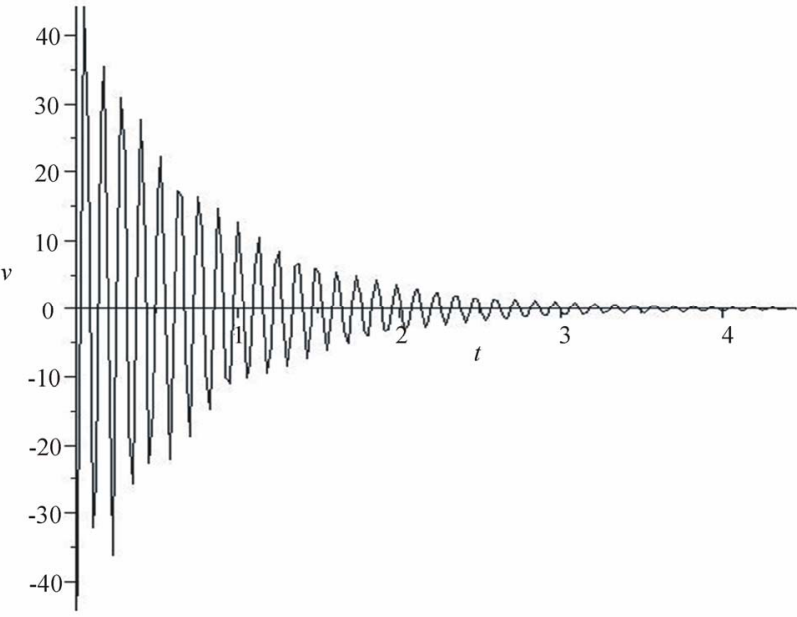

(d)

Figure 2. (a) Perturbation of inventory level; (b) Perturbation of actual production Rate; (c) Perturbation of deterioration rate; (d) Perturbation of desired production Rate.

\section{Conclusions}

In this paper we have discussed the problem of adaptive control of a production inventory system with unknown deterioration rate. The desired production rate and updating rule of deterioration rate are derived from the condition of asymptotic stability about its steady state using Liapunov technique. The demand rate is considered as linear function of both inventory level and actual production rate. Numerical examples are presented to show the system behavior for a set of parameter values. It was found that the actual production rate exponentially tends to its goal value while the inventory level, deterioration rate, and desired production rate are damping oscillate about their goal values.

\section{References}

[1] S. Sethi and G. Thompson, "Optimal Control Theory: Appli-Cations to Management Science and Economics,” 2nd Edition, Kluwer Academic Publishers, Dordrecht, 2000.

[2] I. Dobos, "Optimal Production-Inventory Strategies for a HMMS-type Reverse Logistics System,” International Journal of Production Economics, Vol. 81-82, 2003, pp. 351-360. doi:10.1016/S0925-5273(02)00277-3

[3] S. Nahmias, "Perishable Inventory Theory: A Review," Operations Research, Vol. 30, No. 3, 1982, pp. 680-708. doi:10.1287/opre.30.4.680

[4] B. Porter and F. Taylor, "Modal Control of Production-Inventory Systems," International Journal of Systems Science, Vol. 3, No. 3, 1972, pp. 325-331. doi:10.1080/00207727208920270

[5] F. Raafat, "Survey of Literature on Continuously Dete- riorating Inventory Models,” Journal of the Operational Research Society, Vol. 42, 1991, pp. 27-37. doi: $10.2307 / 2582993$

[6] E. Khemlnitsky and Y. Gerchak, "Optimal Control Approach to Production Systems with Inventory Level Dependent Demand," IIE Transactions on Automatic Control, Vol. 47, No. 3, 2002, pp. 289-292. doi:10.1109/9.983360

[7] W. Caldwell, "Control System with Automatic Response Adjustment,” US Patent No. 2,517,081., Filed 25, 1950.

[8] J. Aseltine, A. R. Mancini and C. W. Sartune, "A Survey of Adaptive Control Systems," IRE Transactions on Automatic Control, Vol. 3, No. 6, 1958, pp. 102-108. doi:10.1109/TAC.1958.1105168

[9] I. D. Landau, “Adaptive Control: The Model Reference Approach,” Marcel Dekker, New York, 1979.

[10] K. S. Narendra and A. M. Annaswamy, "Stable Adaptive Systems,” Prentice-Hall, Englewood Cliffs, 1989.

[11] A. El-Gohary and A. Al-Ruzaiza, "Chaos and Adaptive Control in Two Prey, One Predator System with Nonlinear Feedback," Chaos, Solitons and Fractals, Vol. 34, 2007, pp. 443-453. doi:10.1016/j.chaos.2006.03.101

[12] A. El-Gohary and R. Yassen, “Adaptive Control and Synchronization of a Coupled Dynamo System with Uncertain Parameters," Chaos, Solitons and Fractals, Vol. 29, 2006, pp. 1085-1094. doi:10.1016/j.chaos.2005.08.215

[13] L. Tadj, A. Sarhan and A. El-Gohary, "Optimal Control of an Inventory System with Ameliorating and Deteriorating Items,” Journal of Applied Sciences, Vol. 10, 2008, pp. 243-255.

[14] A. Foul, L. Tadj and R. Hedjar, "Adaptive Control of Inventory Systems with Unknown Deterioration Rate," Journal of King Saud University-Science, in Press, 2011, doi:10.1016/j.jksus.2011.02.001 